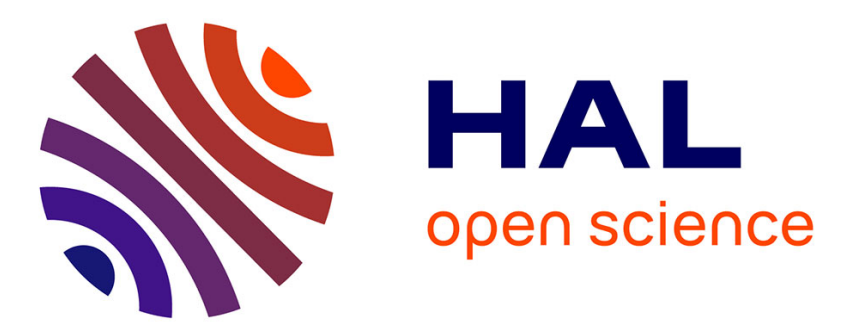

\title{
Céramique laténienne domestique de la région lilloise (Nord)
}

\author{
Germaine Leman-Delerive
}

\section{To cite this version:}

Germaine Leman-Delerive. Céramique laténienne domestique de la région lilloise (Nord). Gallia - Fouilles et monuments archéologiques en France métropolitaine, 1984, 42 (1), pp.79-95. 10.3406/galia.1984.1911 . hal-01940990

\section{HAL Id: hal-01940990 \\ https://hal.science/hal-01940990}

Submitted on 6 Feb 2020

HAL is a multi-disciplinary open access archive for the deposit and dissemination of scientific research documents, whether they are published or not. The documents may come from teaching and research institutions in France or abroad, or from public or private research centers.
L'archive ouverte pluridisciplinaire HAL, est destinée au dépôt et à la diffusion de documents scientifiques de niveau recherche, publiés ou non, émanant des établissements d'enseignement et de recherche français ou étrangers, des laboratoires publics ou privés.

\section{(ㅇ)(1) $\$$}

Distributed under a Creative Commons Attribution - NonCommercial - NoDerivatives $\mid 4.0$ 


\title{
CÉRAMIQUE LATÉNIENNE DOMESTIQUE DE LA RÉGION LILLOISE (Nord)
}

\author{
par Germaine LEMAN-DELERIVE
}

Cette céramique provient exclusivement d'habitats récemment découverts grâce aux prospections de H. Halhaut ${ }^{1}$. Comme dans le reste de la région Nord-Pas-de-Calais, ceux-ci se localisent le long des cours d'rau, dans les fonds de vallées souvent fangeuses de la Haute-1)eule et de son affluent la Varque (fig. 1). Les nécropoles correspondantes sont tout à fait inconnues. L interèt de ces prospections est double, d'une part en ce qui concerne la topographie des sites habités et l'étude des structures, domaines qui feront l'objet d'une autre étude, et d'autre part, dans l'élaboration d'une typologie de la céramique qu'on voudrait tenter d'aborder ici. En effet, la proximité des différents lieux de découverte, l'abondance relative des formes à l'intérieur de chaque ensemble sont autant d'éléments qui facilitent les comparaisons et qui, par conséquent, permettront peut-être de repérer les premieres caractéristiques de l'écolution des différents faciès en céramique.

Les ensembles les plus intéressants furent trouvés à Houplin-Ancoisne, Seclin et Loos (2 sites). On ne peut isoler toutefois aes découvertes des documents particulièrement nombreux en provenaner de Villeneuve-d'Ascep. S'il est encore prématuré de publier le résultat de cette fouille actuellement en cours, il conviendra de l'utiliser à titre de comparaison.

1. Houplin-Ancoisne (fig. 2 à 6 ). En arril 1977, au lieu dit Marais d'Ancoisne, la pose d'une canalisation permit à H. Lalbaut te repérage d'une large structure d'époque laténienne'2. Le nettoyage rapide de la coupe mit en évidence une rouche tourbeuse, longue d'une dizaine de mètres. profonde de près d'un mètre. En dépit du peu de temps imparti, il fut possible de recueillir un lot important de céramiques, de silex, et d'ossements d'animaux, attestant la présence indéniable d'un habitat à cet endroit.

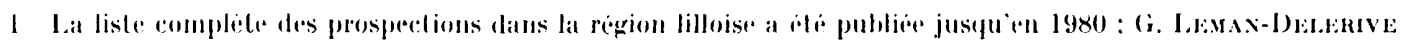
et (i. I.efrave, Forteresses ganloises el gisements de l'dige du fer dans le Vurdl'as-de-falais, 2 vol., Allas Archéologique

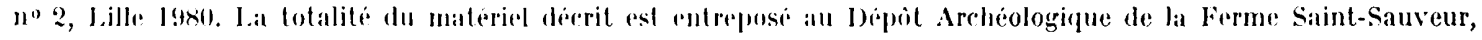
avenue du Bois, 59650) Villeneuve d'Ascy.

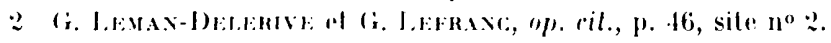




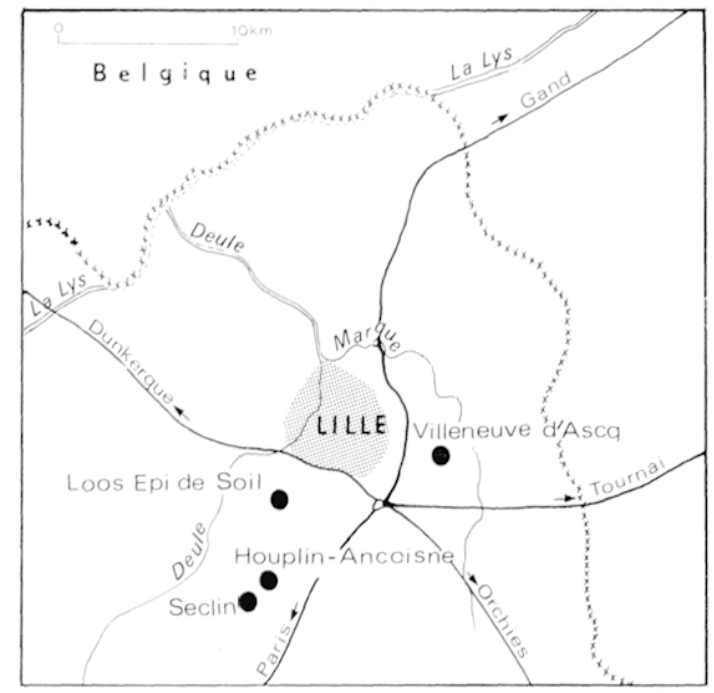

1 Localisation des différents sites de l'agglomération lilloise.

Parmi les quelques centaines de tessons, il convient de distinguer deux grands groupes : une céramique fine, lissée, d'aspect savonneux et une céramique plus grossière et rugueuse au toucher. Mais il faut remarquer qu'une mème forme peut se trouver sous ces deux aspects, comme la situle. Par ailleurs, il est difficile de faire un compte mème approximatif de la répartition des tessons : le morcellement des décors géométriques rend difficile leur attribution à un scul vase; l'irrégularité de certains fragments rugueux amène à douter parfois de l'existence de plusicurs formes. De plus. la fouille n'a pas été, de loin, exhaustive. Par voie de conséquenere, on ne peut que suggeerer une plus faible proportion de la céramique fine par rapport à la céramique rugueuse.

La céramique lissée. . 1) Coupes ou écuelles carénées (figr. 2, 2 a 11). Cette forme est fréquemment représentée parmi la céramique fine. La hauteur du col est presque constante tandis que la profondeur de la panse est variable. La livre est constituée d'un simple élargissement de la paroi ou d'une baguelte légrement arrondie ou aplatie. Aucun fond n'a pu ètre retrouvé. Comme on le constate souvent, cette forme n'est pas ornée en céramique fine ${ }^{3}$.

2) Situles : ce type doit figurer en plusieurs exemplaires mais aucun n'est complet en céramique fine. Le fond de vase $n^{0} 19$, fig. 3 particulierement soigné comporte deux sillons parallèles dans la partie inférieure de la panse, tandis que des chevrons emboités en oblique, réalisés en pointillés, couvrent la partie centrale. Ce jeu de sillons parallèles figure également sur certains cols (fig. 2, no 12).

3) Vases carénés : ce sont les plus morcelés mais aussi les plus caractéristiques par leur ornementation. Lne dizaine de tessons présentent en effet un décor gravé géométriquement (fig. $4, n^{\text {os }} 22$ à 32). Le motif le plus typique est la mét ope a l'intéricur de laquelle sont tracces des diagronales simples (fig. $4 n^{0} 29$ ) ou doubles (fig. $4 n^{0} 22$ ). La surface délimitée par des lignes gravées horizontalement peut être occupée par des obliques (fig. 4, nos 23. 25) et 26). La partie inférieure de la panse n'est pas décorée mais seulement soulignée d'un sillon dans le vase soigneusement lissé (figr. 3, no 18).

3 P. Rocatrit, In village gauluis de la Tène I a au lieu-dit Entre les denx voies, à Hauviné (Ardennes). Fouilles et collection L. Simonel, dans Memoires de la Sosiete d'Agricullure, Commerce, Sciences el Arts du département de la Marne, l.XXXVIII, 1973, p. 7 à 18. 


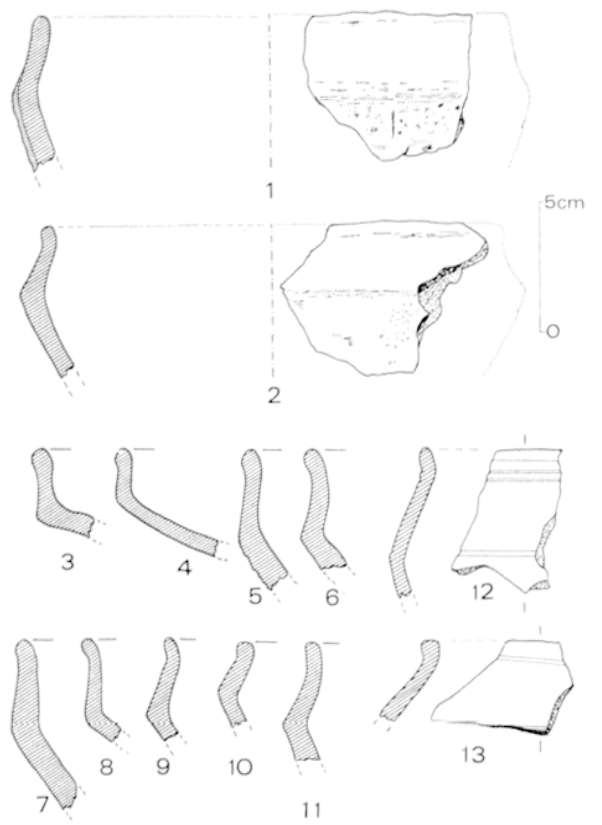

2 Houplin-Ancoisne. 1 à 11 : coupes carénées.

3 Houplin-Ancoisne. Situles et formes apparentées, nos 14 à 21 .
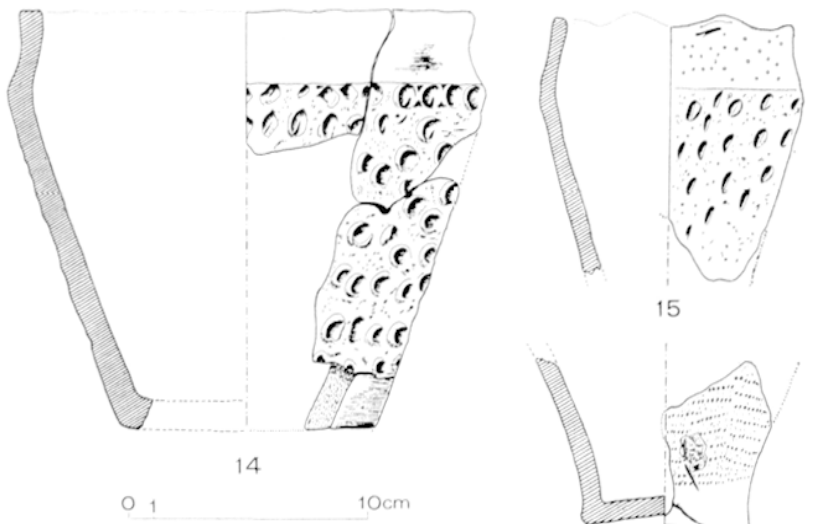

15
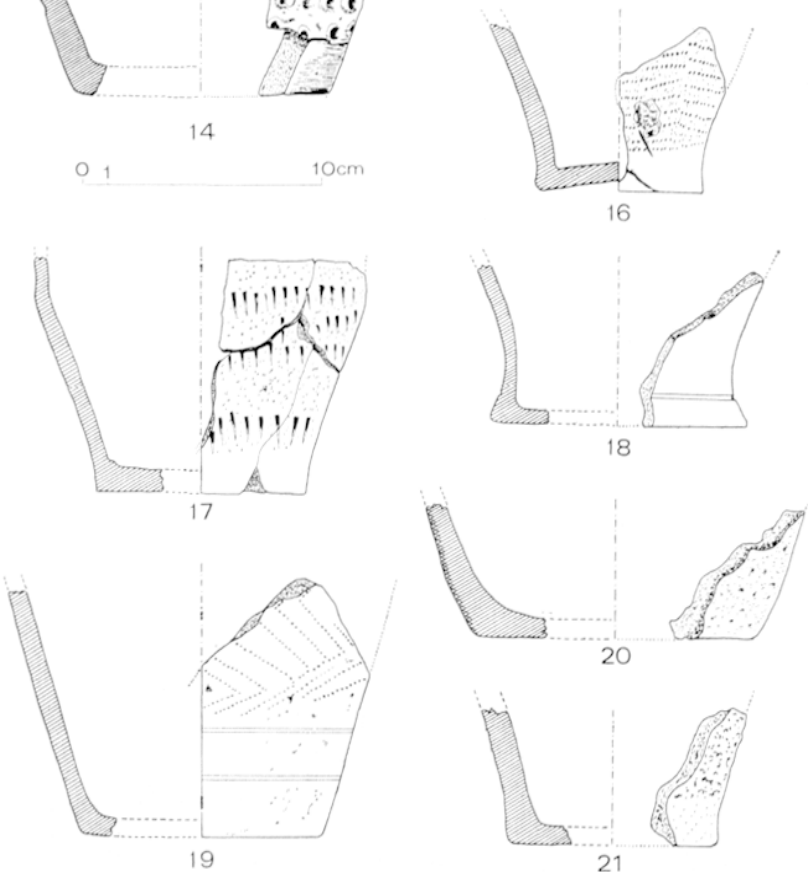

4) Cratère : le fragrment fig. $6, n^{\circ} 54$, avec marli, témoigne sans doute de l'existence d'un exemplaire de grand vase appelé parfois cratère.

La céramique rugueuse. - 1) Silules : sur l'exemplaire le plus complet (fig. 3, $\mathrm{n}^{0}$ 14) la panse tronconique comporte des rangs plus ou moins réguliers d'impressions au doigt. Le pied, à fond plat, n'est pas décoré mais seulement lissé de même que le petit col à la lèvre simplement constituée par un épaississement de la paroi. La partie inférieure conservée (fig. 3, no 17) présente trois ou quatre rangs d'impressions triangulaires réalisées au bâtonnet. Parfois aussi, la panse est couverte "à l'éclaboussé " (fig. 3, nos 20, 21). Le vase sans lévre délimitée mais avec un bord ondulé (fig. $3, \mathrm{n}^{0} 15$ ) peut être également considéré comme un dérivé de la forme en situle.

2) Grandes coupes évasées : deux ensembles de tessons permettent de caractériser un type bien particulier en céramique très grossière : le premier (fig. $\overline{5}, \mathrm{n}^{0} 34$ ) retient l'attention par l'épaisseur de la paroi, la totalité de la couverture en fortes et irrégulières impressions faites au doigt; le bord a été obtenu par une simple égalisation de la paroi, de telle sorte que la pâte s'est repliée parfois vers l'extérieur.

L'autre forme analogue est moins évasée; sa paroi est plus fine et décorée à l'éclaboussé (fig. $\left.\overline{5}, n^{0} 35\right)$.

3) Coupes carénées (fig. 2. $n^{0} 1$, fig. 5, $n^{0} 36$ ), réalisées en céramique rugueuse (la panse est décorée à l'éclaboussé) ; elles présentent une carène légèrement moins anguleuse que celle qui a été rencontrée en céramique fine. 

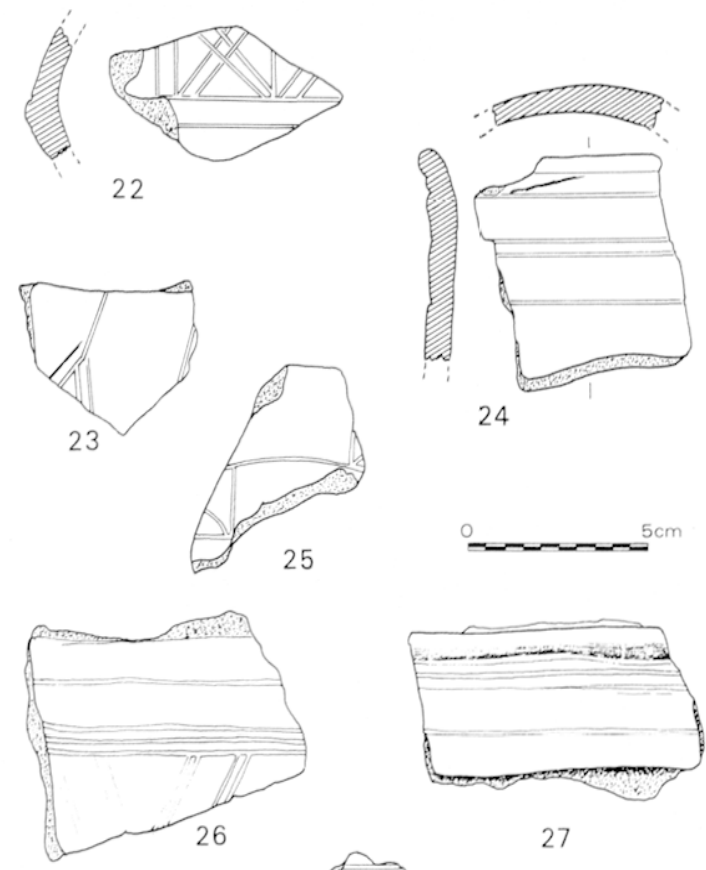

27

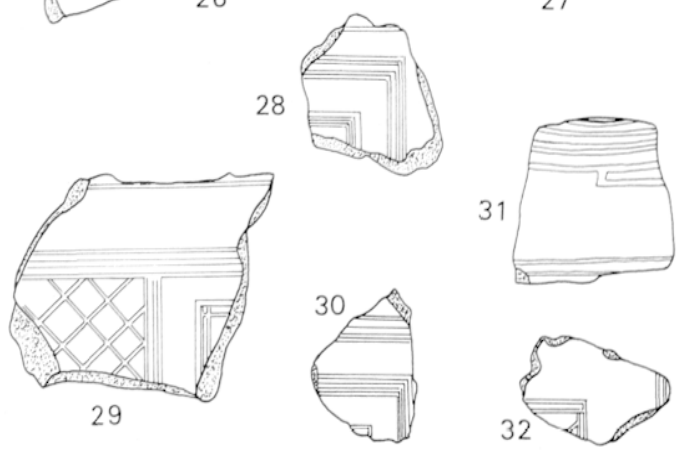

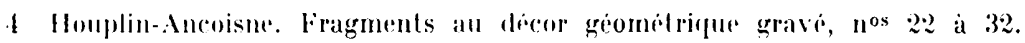

4) (irand bassin : un bassin à panse arrondie, trailée à l'éclaboussé, présente une livre épaisse et évasée. Le col est grossièrement lissé (fig. 5, nº :33).

Autres fragments de bords. Lin grand nombre de fragments de bords ont été découverts. Il est difficile de les répartir en céramique lissée et céramique ruqueuse, puisque mème en céramique grossière, le bord est souvent lissé. L attribution à une forme définie est éqalement téméraire. Certains fragments semblent façonnés pour recevoir un couvercle (lig. $6, n^{0 s} 47$ et 48 ).

Dalation. La determination de la chronologie se trouve facilitere par la publication de fouilles récentes d'habitat.

Certes, la céramique fine, bien connue, constitue une base de référence aisée puisquelle trouve son équivalent dans la céramique funéraire champenoise. A l'inverse, la céramique rugueuse grossière napparait que dans les habitats et seule son asssociation avec de la céramique fine permet d'inférer une chronologie.

Quelques types de céramique ruguense peuvent toutrfois desormais se délinir : la situle se retrouve sur le sile d'Orp-le-Grand (Brabant Belge), ornér de stries curvilignes 


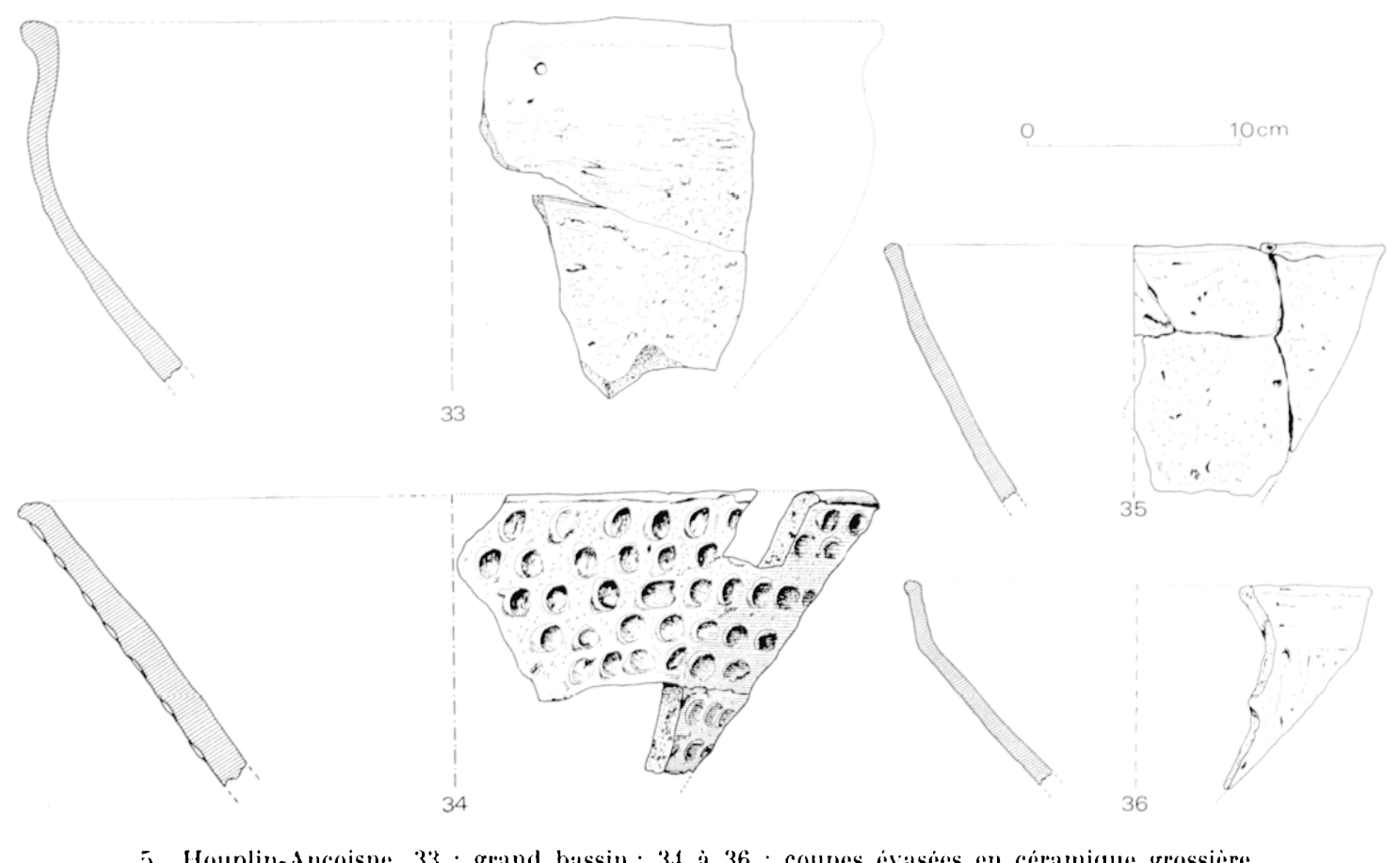

5 Houplin-Ancoisne. 33 : grand bassin ; 34 à 36 : coupes évasèes en céramique grossière.

incisées au peigne ${ }^{4}$. Très fréquent également dlans les habitats est le grand vase à épaulement souligné d'impressions et à petit bord évasé ; il figure à Chassemy ${ }^{5}$, à Menneville ${ }^{6}$ et à Villeneuve-Saint-Germain ${ }^{7}$ en ce qui concerne le domaine marnien, ou encore à Spiennes

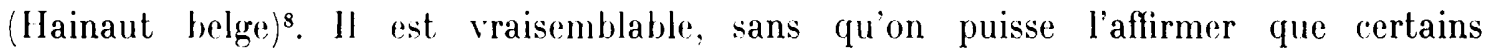
fragments d'Houplin-Anecoisne appartiennent à ce lype (fig. 6, nos 49, 52, 53, 60-63) quoiqu'ils ne présentent pas de cupules digitales sur le borl, qui affecte ainsi un aspect crénelé. Cne troisième forme caractéristique est la grande coupe évasée sans col. qu'on retrouve a llauviné. Par ailleurs, manque à Houplin-Ancoisne la coupe à bords ourlés, si typiqurito.

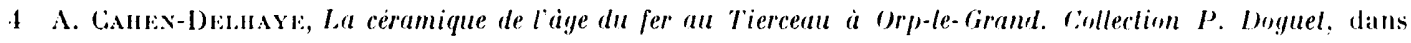
Répertoires archéologiques, série B, Bruxelles 197.t, fig. 12, no 137. On peut consulter igalement sur cr site, A. Cant:x

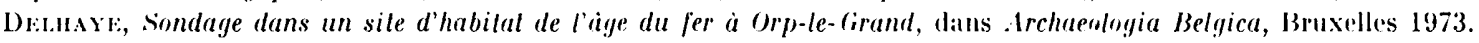

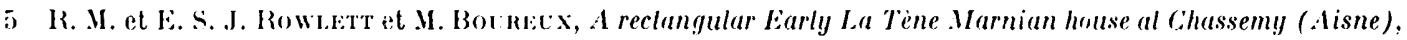
dins IVorld Archueslog!t, I, 1969, p. 106 a 135, cf. fig. 33.

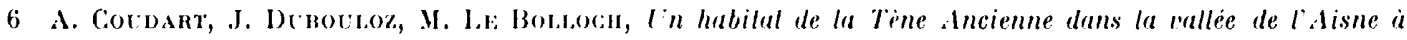
Menneville (Aisne), dans l'Age du fer en France seplentrionale, Vémoires de la sociele Archeologique Champenoise, :2, 1981 , p. 1:21 a 130, cf. fig. 7 .

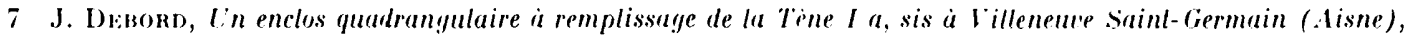
ibid., p. 107 a 1:20, cf. fig. 10 : no $7 \times$ E 07012 ,

$\checkmark$ Spiennes, Camp i Cayaux, fond F; rf. M. E. Mantex, La periode de la Tine en Belgique. I.e Groupe de la IIaine, Bruxelles 1961, p. 1:2x, fig. $53, \mathrm{n}^{\circ} 3$.

9 P. Rotalet, "p. cit., fig. $\mathrm{V}, \mathrm{n}^{\circ} 11$.

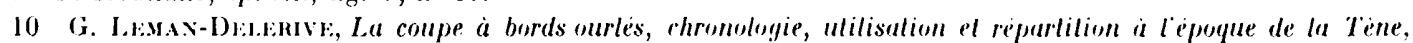
dans l'Antiquite classique, XI.II, 1973, p. 406 a 426 . Voir la derniere mise au point, a part ir de la documentation tress ilargie depuis 10 ans, dans la these de X. Frems, The Early Iron dge in the Paris Bassin-Hallstall r: and I), Oxford, 1982,1, p. $156-15 x$. 

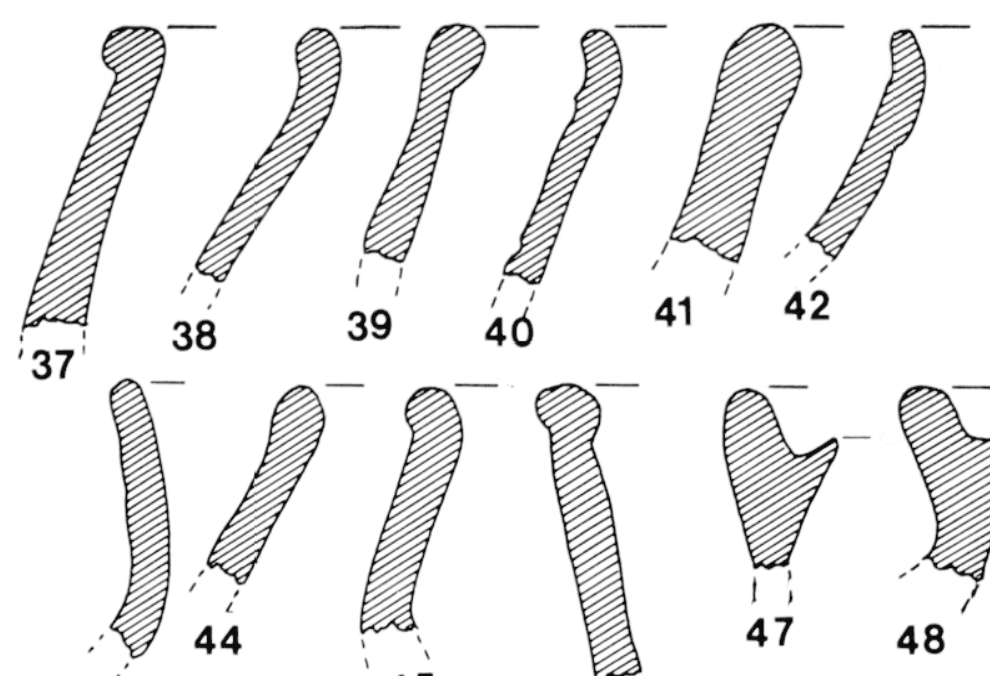

43
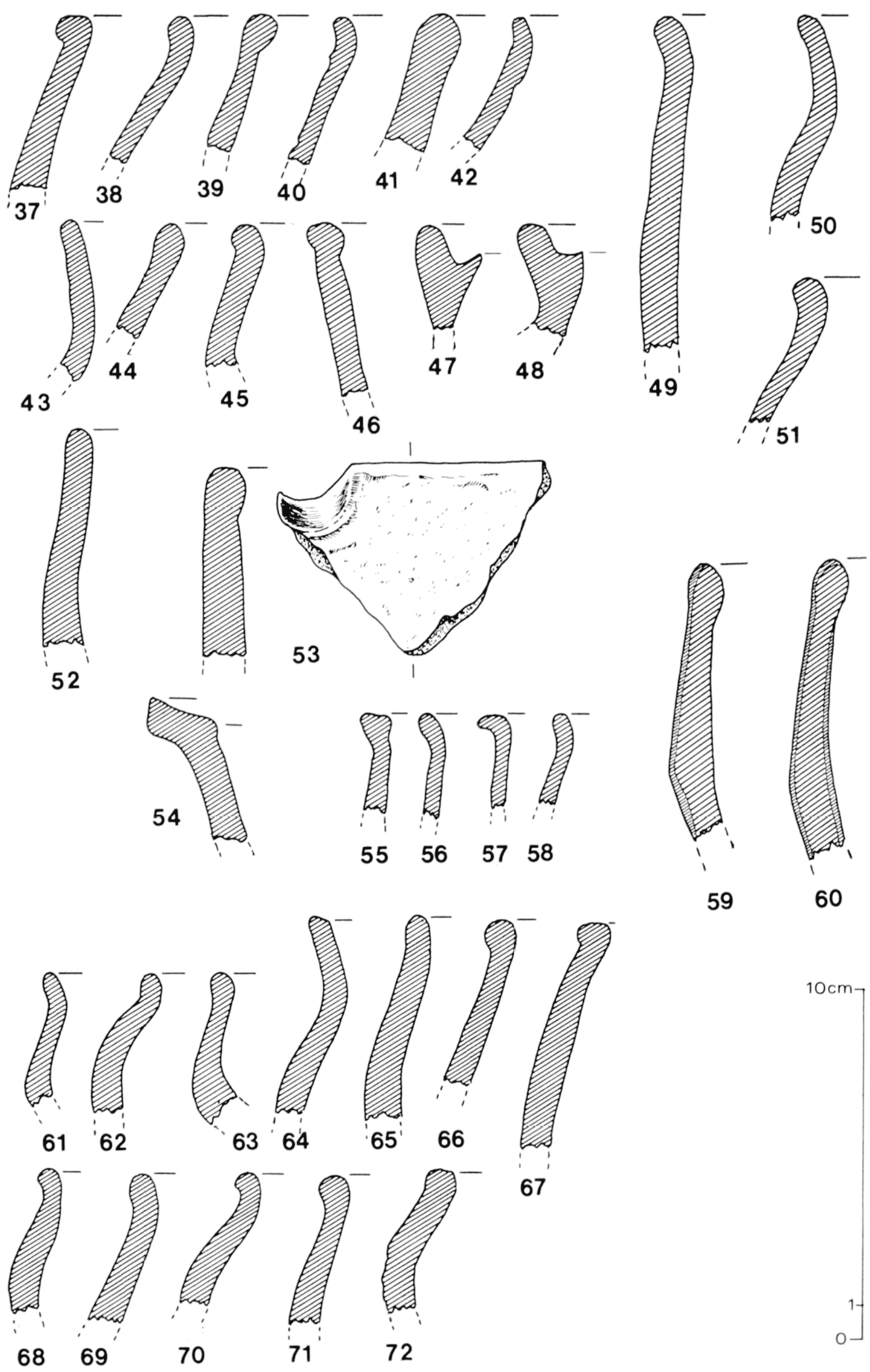

59

6 Houplin-Ancoisne. Différents bords, nos 37 à 72 . 
Les différentes formes de céramique rugueuse citées plus haut se trouvent associées, comme à Houplin, à de la céramique fine, à décor géométrique, attribuéc par des références funéraires au début de La Tène I" . Par conséquent, il est permis de proposer que l'habitat d'Houplin-Ancoisne appartient également à la phase I a de La Tène.

II. Loos I (fig. 7, 8 et 9). En 1974, dans l'exploitation d'une argilière au lieu-dit l'Epi de Soil, une fosse fut fouillée en sauvetage. De forme irrégulière, sans stratification, elle contenait un lot considérable de tessons dans un volume fort réduit. Cette fosse étant vraisemblablement un silo, son remplissage homogène permet de considérer avec certitude cet ensemble de céramique comme fermé, ce qui en accroît l'intérêt ${ }^{12}$.

Ces céramiques, comme celles d'Houplin-Ancoisne, offrent une surface grise ou gris noirâtre, avec dégraissant apparent. Les formes hautes dominent en nombres d'exemplaires.

Parmi les trois vases de forme basse, une grande coupe à épaulement légèrement arrondi et à petit col concave présente une panse ornée de doubles stries approximativement verticales et parallèles. Deux trous ont été perçés à travers le col (fig. $7, \mathrm{n}^{0} 1$ ). Dans la seconde coupe (fig. $7, \mathrm{n}^{\circ} 2$ ), l'épaule est plus anguleuse et le col est proportionnellement plus important et plus évasé. Toutefois, le décor de la panse, comparable à celui de l'exemplaire précédent, permet de supposer l'identité de l'atelier.

La troisième forme basse est une coupe à épaule légèrement carénée, sans décor (fig. $7, n^{\circ} 3$ ), en céramique épaisse plus soigneusement lissée que les deux exemplaires précédents.

Deux types essentiels sont à distinguer parmi les formes hautes. En premier lieu, une forme apparentée à la situle, à fond plat, épaule légèrement arrondie. Sur l'exemplaire le plus complet (fig. $7, \mathrm{n}^{0} 4$ ), le col est de proportion plus importante, par rapport à la hauteur totale, que dans les exemples plus anciens d'Houplin-Ancoisne; il s'incurve légèrement, la lèvre étant évasée et arrondie. L'ornementation se limite à la panse, garnie de petites impressions très irrégulièrement réparties. A l'inverse, le décor est particulièrement soigné dans l'exemplaire $\mathrm{n}^{0} \overline{5}$, où la panse comporte des chevrons délimitant des zones alternativement lissées ou remplies de pointillés réalisés en creux. Le col de ce vase est séparé de l'épaule et de la lèvre par un jeu de sillons. Deux autres vases fragmentaires peuvent être rapprochés, par leur forme générale, de la situle ; l'un (fig. $7, n^{0} 6$ ) est caractérisé par une ligne d'impressions de forme ronde située à la limite du col et de la panse travaillée à l'éclaboussé ; le second (fig. $7, n^{0} 8$ ) est un fond dont la panse entière est travaillée à coup d'ongle. Quant au petit vase $\mathrm{n}^{0} 7$, il est difficile de le rattacher à une catégorie précise. On pourrait peut-être le considérer comme une réduction du type de loin le plus représenté dans la fosse de Loos, qui est un grand vase à la fonction purement domestique.

Ce deuxième type se caractérise par son ornementation limitée à un rang d'impressions au niveau de l'épaule, par un traitement de la panse à l'éclaboussé. De facture très grossière, il comporte un fond plat, un épaulement parfois très arrondi. Le col lissé peut être constitué

11 Voir également le site du Mont Kemmel (Belgique) : $\Lambda$. vax Doonselatr, Ler Kemmelberg, ein Keltischer Herrensitz ?, dans Alba Régia, Annales Musei Stéphani Régis XIV, 1975, p. 79-84.

12 G. Lemax-1) HLentye el G. I.firaxc, 1980, op. cit., p. 49, site no 1. 

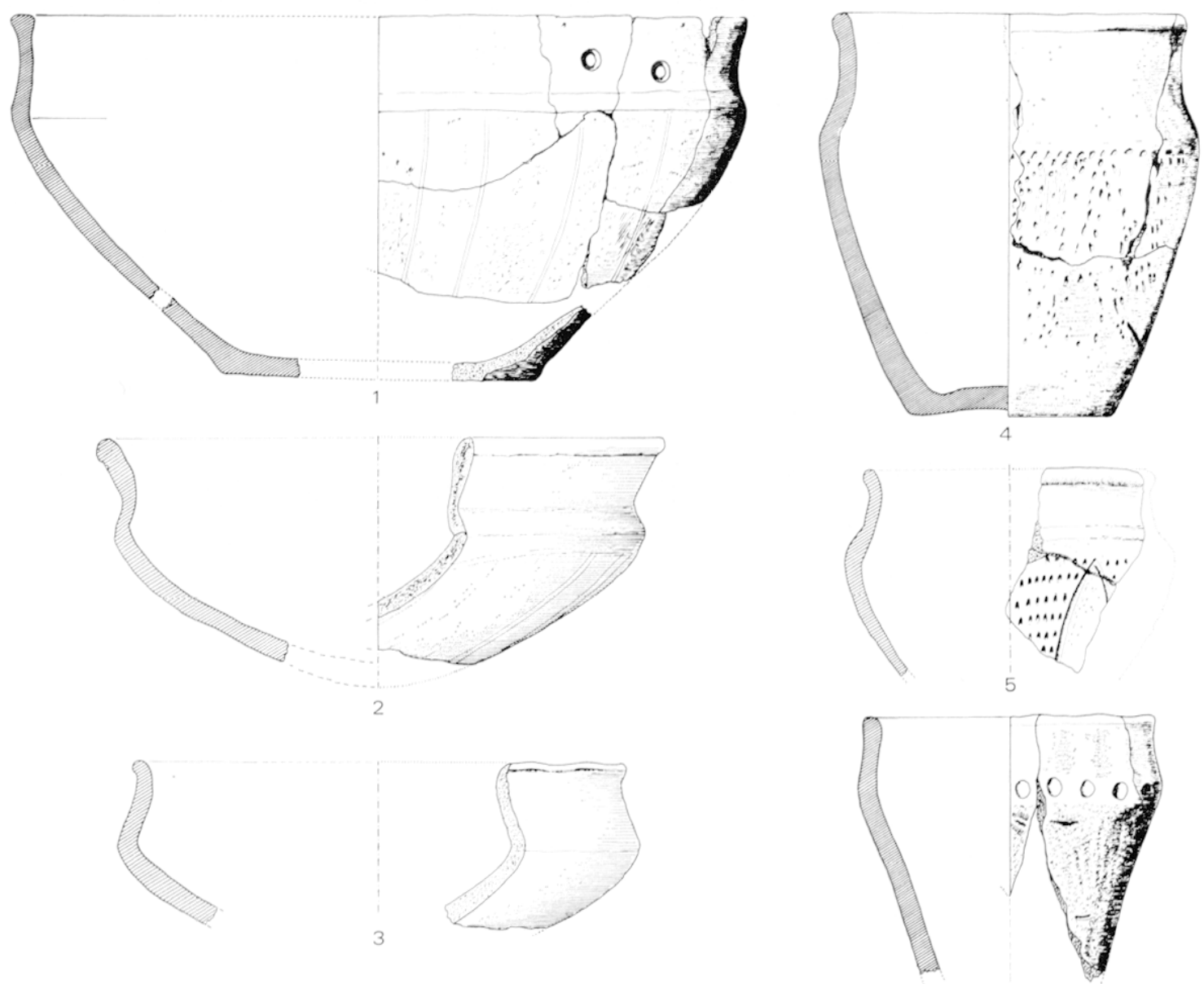

6
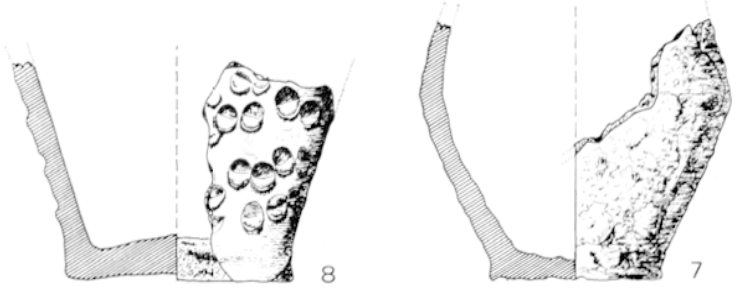

7 Loos 1.1 , 2 : coupes à panse décorée; 3 : coupe sans decor à epaule légerement carrine ; 4 a $x$ : formes apparenties al la silulte.

d'un simple épaississement de la paroi, ou s'évaser plus ou moins fortement (fig. 8 et 9, nos 9 à 23). Les cupules qui délimitent l'épaule sont réalisées à l'ongle, au doigt, ou au bâtonnet. Leur régularité est variable suivant les vases. La panse d'un exemplaire a été totalement couverte par cette technique rudimentaire. Ieux autres tessons furent peut-être lissés à l'herbe, puisqu'ils présentent sur la panse des stries affectant des directions obliques ou horizontales.

Quelques tessons attestent lexistence de vases a épaule llanquere de bourrelet ou souligné de sillons horizontaux ou obliques, an modelé assez, fin et soigné (fig. 9, nos 2-4-26). 

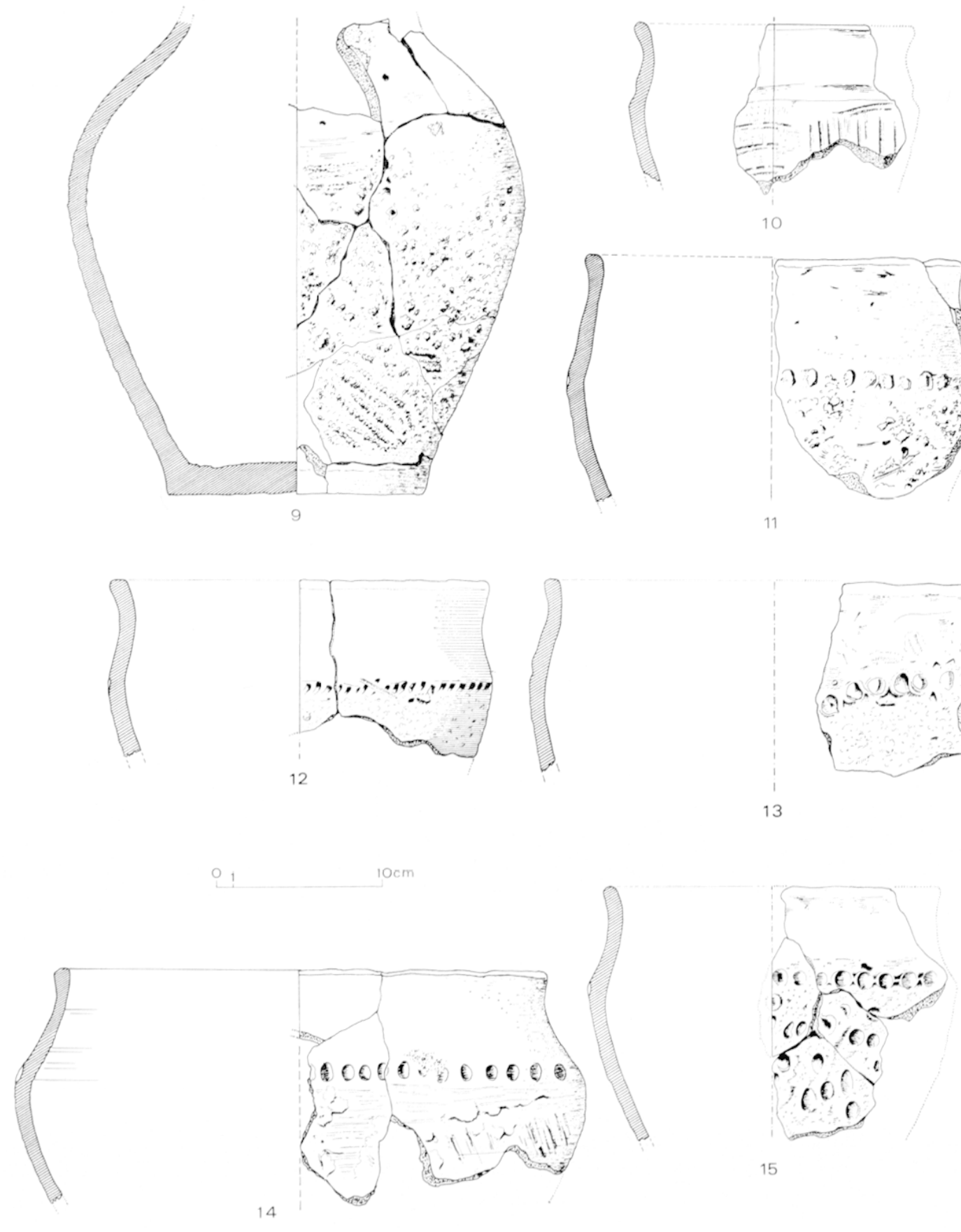

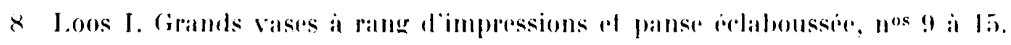

Dalation. - La chronologie s'appuie une nouvelle fois sur le matériel funéraire. En effet, il semble que les fouilles d'habitat ayant fourni un matériel analogue ne se sont pas multipliées ces dernières années.

Deux fouilles anciennes ont fourni des pièces de comparaison. Dans le "Groupe de la Haine", on peut sans doute considérer comme contemporain de celui de Loos le matériel 

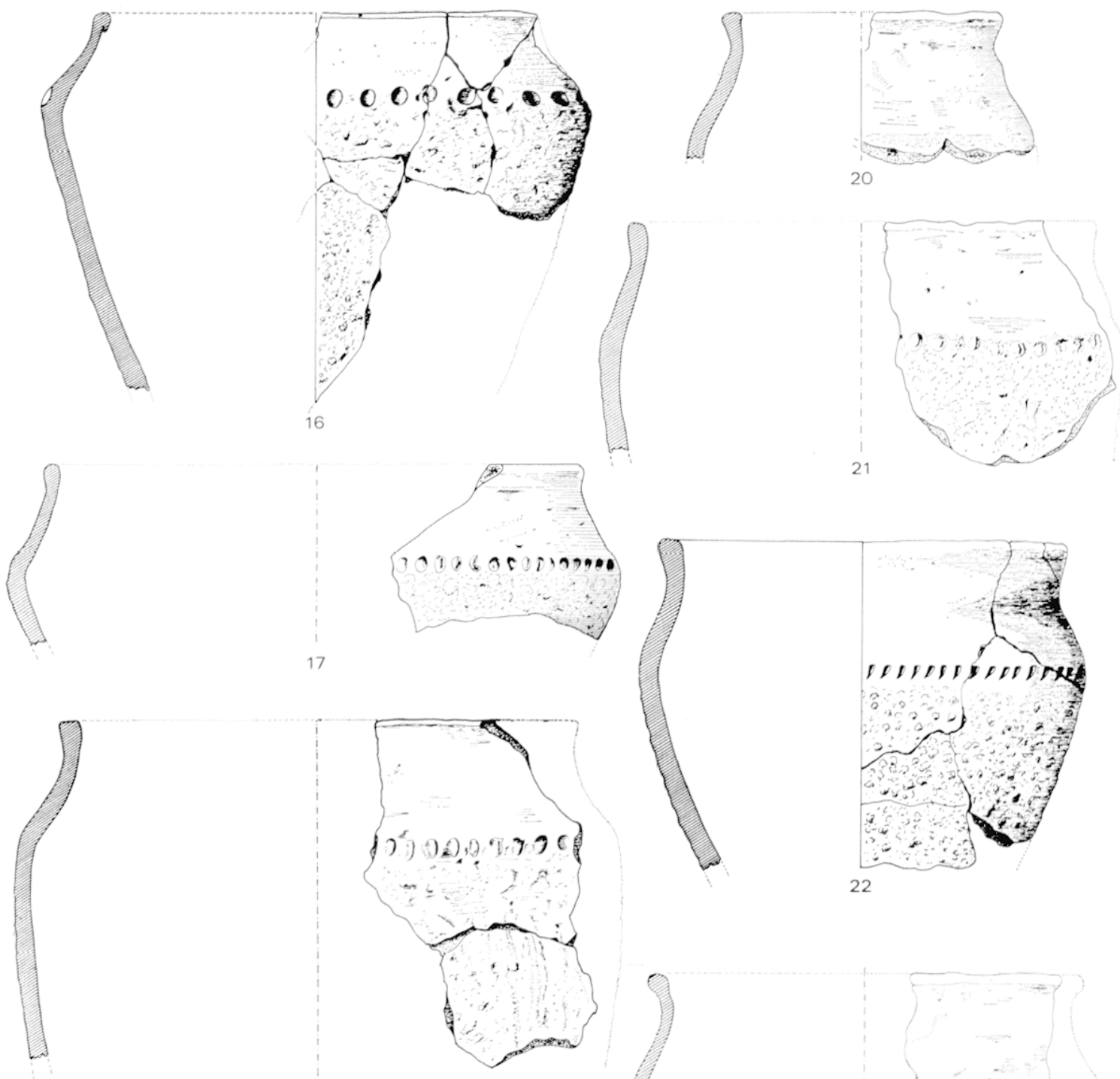

18
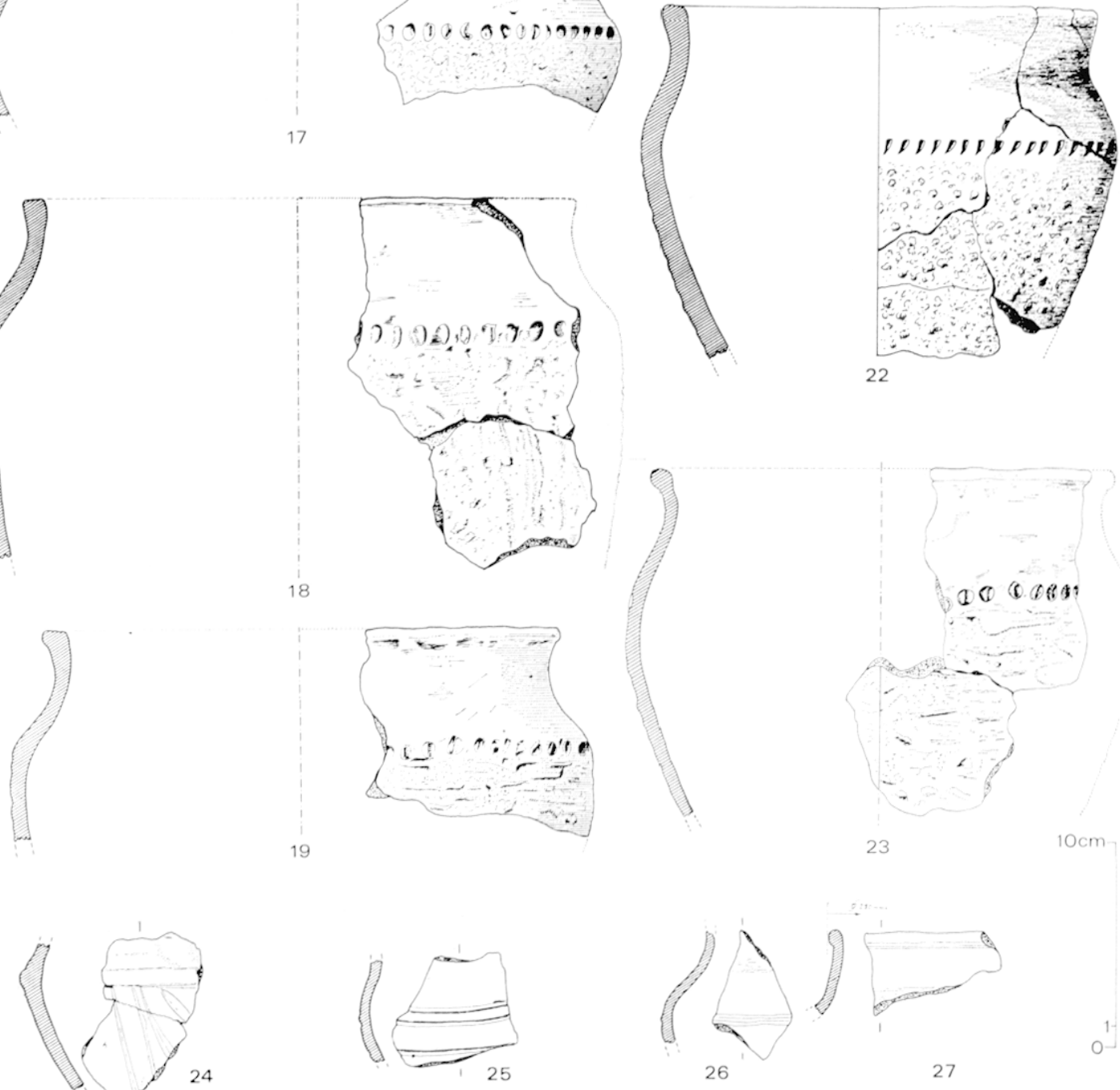

24

9 Loos I. Grands vases à rang d'impressions et panse éclaboussée, nos 16 à 23 ; Vases en céramique assez fine et épaule flanquée de bourrelet: nos 24 à 27. 

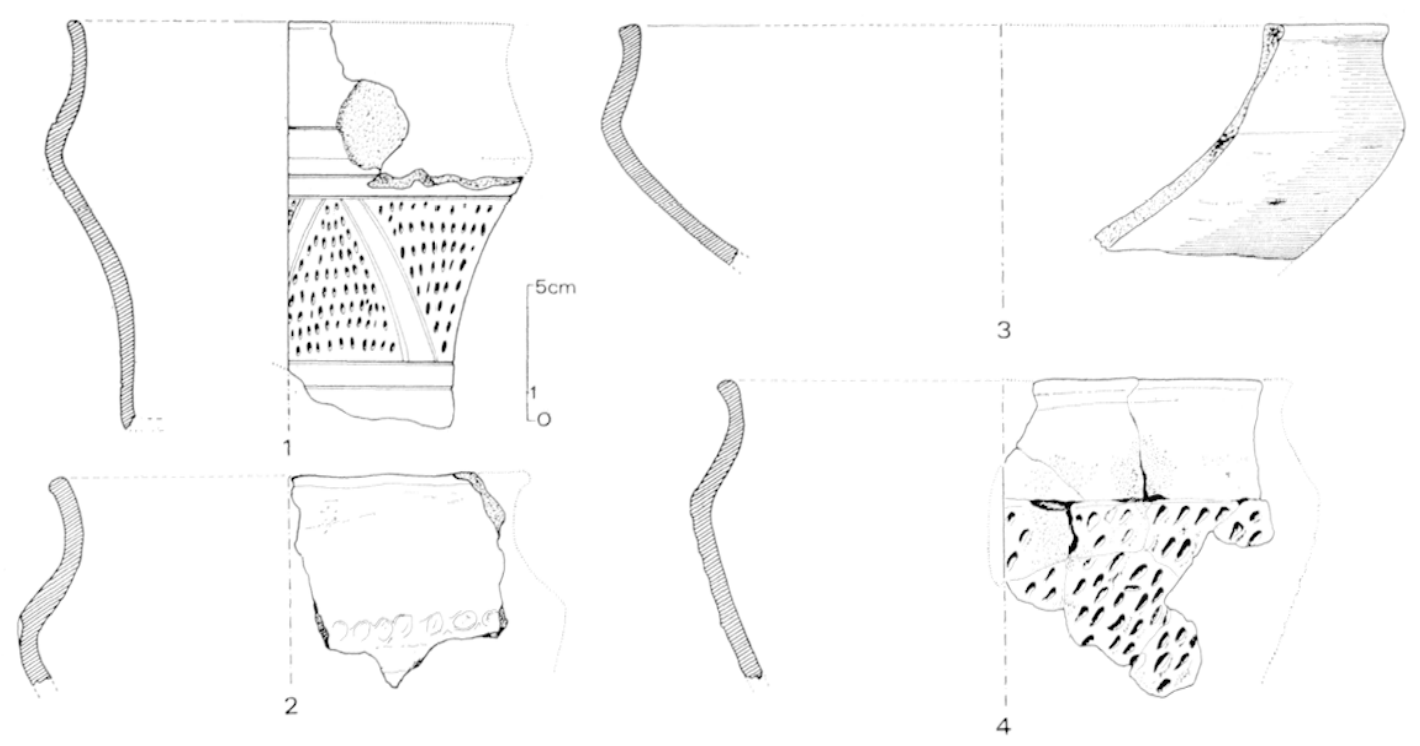

10 Villeneuve d'Aseq, Les Prés. Campagne 1979, ensemble de la Téne moyenne.

de la "hutte A » de Spiennes ${ }^{13}$, comme les coupes et surtout les vases à provision avec paroi éclaboussée. Le Musée d'Epernay conserve également un ensemble assez proche provenant d'Hauviné, lieu-dit Armel et Hurteaux (Collection Simonnet) ${ }^{14}$. Mais, dans la région lilloise, le mobilier de l'Epi-de-Soil trouve des comparaisons nombreuses dans le site de Villeneuved'Ascq, au lieu-dit les Prés. La partie nord du site, fouillée en 1979, était essentiellement constituée d'un ensemble de grands dépotoirs juxtaposés et riches en céramiques. Ciertaines formes et certains décors sont tout à fait comparables à ceux de Loos, en particulier la coupe à épaule légèrement carénée et le décor en triangle avec zones de pointillés (fig. 10).

En céramique funéraire, le décor de triangle ou de chevron (qui peut se limiter à une zone de la panse) apparaît fréquemment à Allonville ${ }^{15}$, à Port-le-('irand ${ }^{16}$ ou à Breuil-le-Sec ${ }^{17}$, pour ne citer que les exemples géographiquement les plus proches. Les vases de Caurel (sépulture 587) évoquent également le profil du vase no 1 de Loos ${ }^{18}$.

Ainsi, dans l'état actuel de nos connaissances, la céramique domestique n'offre encore que peu de points de repère, et seules quelques formes de la céramique funéraire sont comparables ; elles sont néanmoins assez typiques pour proposer que la fosse de Loos étudiée ici est à placer au début de La Tène II.

13 M. E. MARIËx, op. cil., fig. 48, p. 104.

14 Inédit. Je remercie vivement $\mathrm{M}$. Roualet de mavoir indiqué l'existence de ce matériel.

15 A. Ferdiere, R. Galdefroy et J. Massy, C. Marmoz, J.-P. Monex, F. Poplix, Les sépullures gauloises d'Allonville (Somme), dans Bulletin de Société Préhistorique Frangaise, LXX, 1973, Eludes el Travaux, p. 479-492, $2 \cdot$ losse.

16 G. Lemax-Delenive, Le cimetière gaulois de Port-le-Grand, Somme, Essai d'interprétation des fouilles de 1833-34 dans Cahiers archéologiques de Picardie, 1976, p. 97-115, cf. p. 100, F. 6 v. 3.

17 A. Duval, Aspects de la Tène moyenne dans le Bassin Parisien, dans Bullelin de la Socièlé Préhistorique Française, 73, 1976, Eludes el Travaux, p. 457 à 484, cf. fig. 14, p. 473.

is A. Dival, ibid., p. 472 , ng. 13. 
III. Seclin (fig. 11). Trouvé dans un petit fossé, au lieu-dit le Pelil Marais fouillé sur quelques mètres seulement en 1978 , ce petit matériel homogène illustre une certaine évolution dans le matériel domestique par rapport au site de Loos précédemment étudié ${ }^{19}$.

La forme la plus représentée est la terrine à col orné. La panse est arrondie, sans décor ; l'épaule peut ètre encore assez anguleuse. Le fond est plat ou très légèrement concave. Mais la caractéristique essentielle de cette forme semble être le décor du col, qui est constitué de sillons parallèles et situé à la hase de la lèvre assez haute, et à la limite de l'épaule; le col affecte aussi un profil incurvé (fig. $11, n^{\text {os }} 1$ à 6 ).

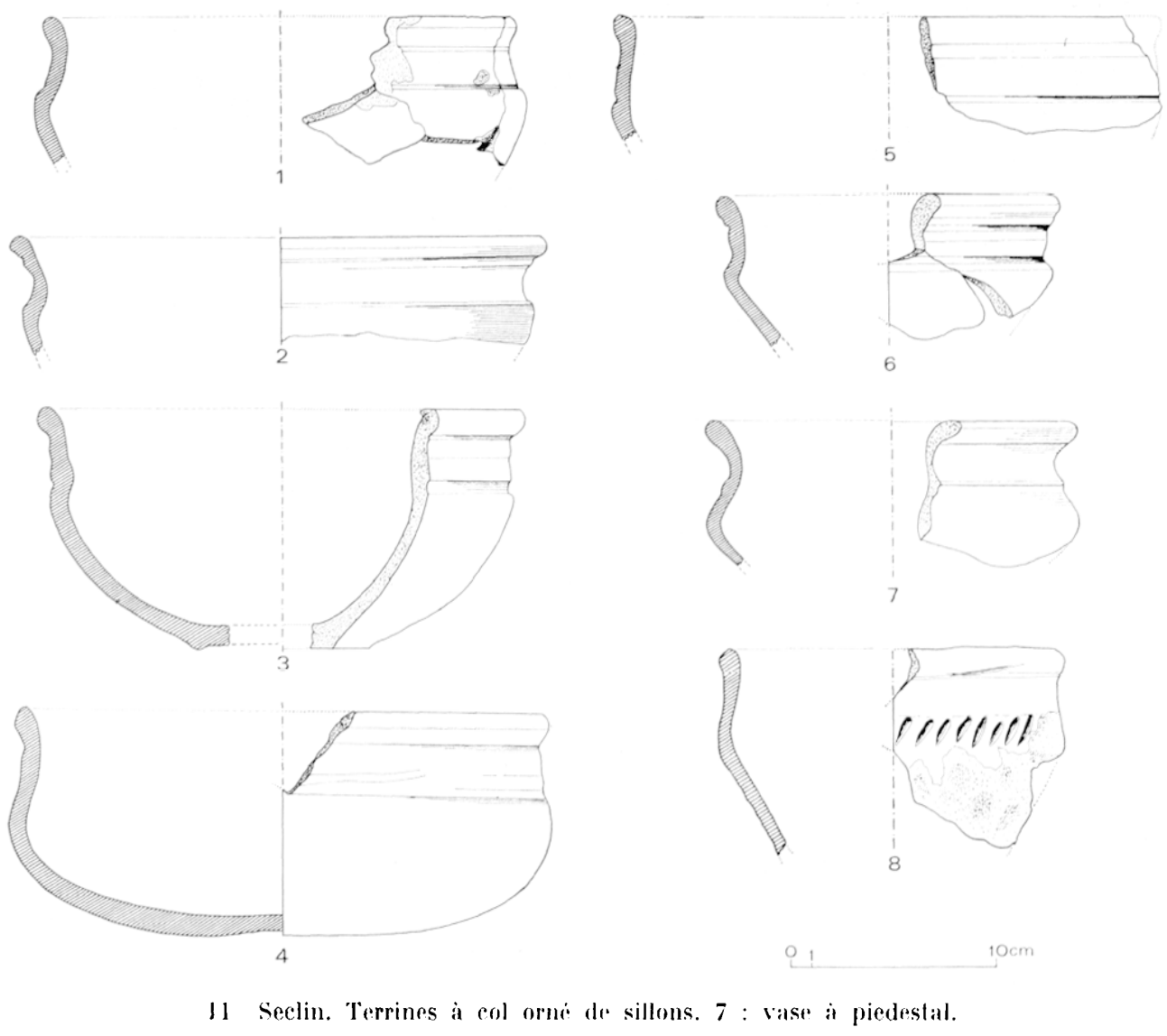

Des deux autres vases, on retiendra surtout le $n^{0} 7$ de la fig. 11, à lèvre évasée et bourrelet médian qu'il faut considérer comme la partie supérieure d'un vase à piedestal du type Marson-Montfercaut, rencontré par exemple à Hauviné (Ardennes), cimetière de Feneux, tombe $26^{20}$.

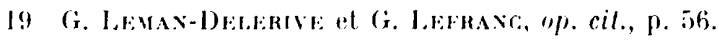

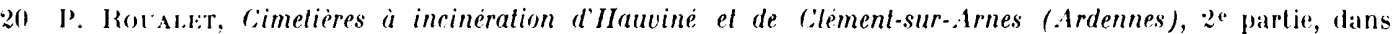
Memoire de la sociele dagricullure, commerce, sciences el arls de la Marne, XCIII, 1978, p. :23-24, of. pl. XII. 
Dalation. -.. L’identification du vase à piédestal amène à chercher des comparaisons dans la période de la Tène moyenne, dont il est l'un des plus caractéristiques.

Il semble, par ailleurs, que les terrines de Seclin présentent un profil analogue à celui des vases des lumuli de la forêt des Pothées (Ardennes). que l'on place généralement à la phase moyenne de La Tène ${ }^{21}$. Faut-il également les rapprocher des exemplaires de La Courle à LevalTrahegnies que X. E. Mariën place à La Tène finale ${ }^{22}$; il sont pourtant associés à un vase haut, ovoïde, avec un décor en zones parallèles remplies de pointillés. et donc très semblable à ceux qu'on rencontre maintenant dans les nécropoles de La Tène moyenne (Breuil-le-Sec, Port-le-(Grand, elc.).

Enfin, si on compare le mobilier de Seclin avec celui de Loos, peut-être est-il permis de préciser encore la chronologie à l'intérieur de La Tène moyenne ; il semble en effet qu'en comparant les formes basses, celles de Loos sont plus archaïques et encore proches de La Tène ancienne. Il convient donc de considérer que le site de Loos se place au début de La Tène II, alors que le fossé fouillé à Seclin fut creusé au milieu ou à la fin de La Tène moyenne.

IV. Loos II (fig. 12 et 13). A quelques centaines de mètres du site de l'Epi de Soil, furent découvertes, à l'occasion d'une construction, des structures de l'âge du fer, en particulier un fossé qui, vidé sur quelques mètres, fournit un ensemble de céramiques fort intéressantes.

On peut y distinguer plusieurs types de vases. Remplissant la fonction de la forme basse, une petite terrine (fig. $12 n^{0} 2$ ) présente un épaulement arrondi. Le col est affecté d'un léger bourrelet en sa partie médiane, la lèvre est arrondie et évasée. Les céramiques 1 et 3 de la même figure présentent également un profil torique du col. Ils sont de facture soignée mais n'ont pas été tournés.

Trois autres vases, de forme haute, sont assez semblables par leur profil général, la panse est très arrondie, le fond plat et étroit (fig. $12, n^{\circ} 5$; fig. $13 \mathrm{n}^{\text {os }} 6$ et 8 ). Un rang d'impressions légères souligne la limite du col à la paroi simplement renforcée au niveau de la lèvre. Le décor est constitué de stries parallèles deux à deux, tracées suivant le profil général du vase (fig. $13, \mathrm{n}^{\circ} 8 ; 12, \mathrm{n}^{0}$ )). C'est une ornementation identique qu'on retrouve sur le vase $\mathrm{n}^{0} 7$ de la fig. 13. Toutefois ce vase comporte une ouverture plus large, la panse étant moins arrondie, comme dans l'exemplaire 4 de la fig. 12.

La surface de cette céramique, comme l'ensemble du matériel présenté, est de couleur gris ou gris noirâtre.

Datalion. - Les comparaisons susceptibles de préciser la position chronologique de ce matériel sont des plus rares.

Si on se réfère à la céramique funéraire, le type de vase à tore apparaît dans la Marne, à Hauviné, lieu-dit La Poterie (tombe 27 en particulier). Toutefois, le décor est là plus affiné puisque les différents tores délimitent des zones ornées de pointillés ou de stries ${ }^{23}$.

21 J. Fromous, Recensement des lumulus de fonilles archènlogiques dans la forèl des l'othées, Ardennes, ibid., XXIX, 1955, p. 1-32.

22 M. E. МаRIË, op. cil., fig. 13, p. 34.

23 P. Rovalet, op. cit., ${ }^{\text {re }}$ partie (XCII, 1977, p. 37 à 53, cf. pl. VIII. 

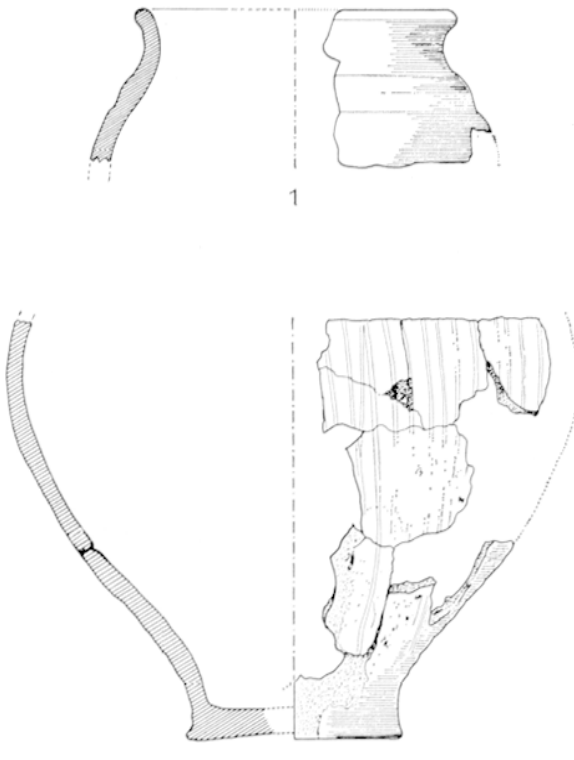

5

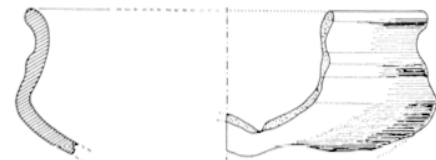

2
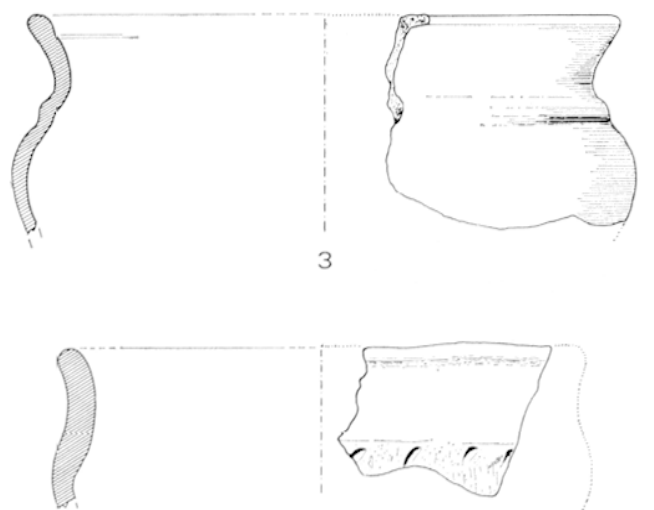

12 Loos II. 1, 2, 3 : vases à profil torique du col; 4 : vase à rang d'impressions et ouverture large ; 5 : vase à panse arrondie, décorée de stries.
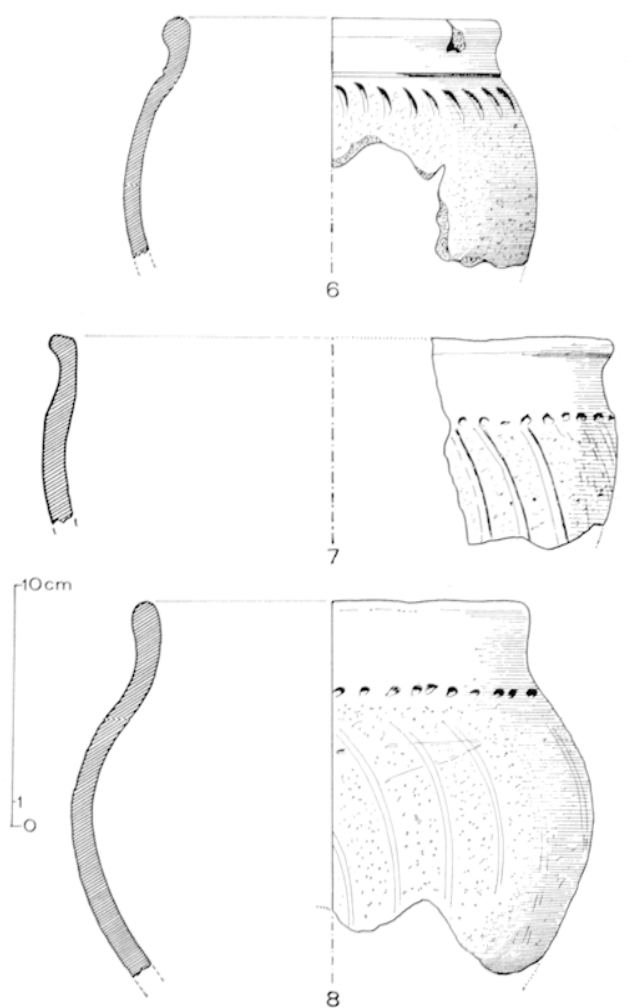

13 L.oos II. Vases à panse arrondie $(7,8 ;$ et décorée de stries. 
En fouille d'habitat, des formes assez proches des vases à panse arrondie ont été trouvées par L. Demarez à Ormeignies (Hainaut belge), mais elles sont encore inédites. La dernière campagne de fouille à Villeneuve-d'Ascq a dégagé une parcelle traversée de réseaux de fossés dans lesquels fut trouvé un matériel proche de celui de Loos (fig. 14).

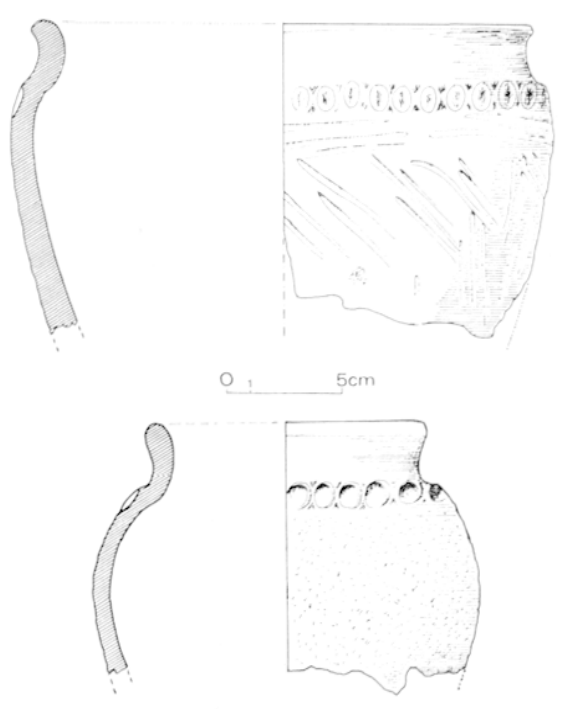

14 Villeneuve d'Ascq, Les Près. Campagne 1982, céramiques du début de la Tène finale.

Dans d'autres fossés, furent exhumés des tessons d'époque augustéenne (tonnelets en terra rubra et nigra). Or l'orientation des fossés augustéens est rigoureusement identique à celle des fossés pré-romains : on serait par conséquent tenté de placer la céramique décrite plus haut immédiatement avant l'époque augustéenne. Ce serait sans doute une conclusion prématurée car les fouilles menées sur les habitats fortifiés datés de l'époque post-césarienne ont produit une céramique beaucoup plus fine, avec une ouverture plus étroite, qu'on ne: peut rapprocher des vases de Loos et de Villencure-d'Ascy ${ }^{24}$. Par ailleurs, les quelques rapprochements possibles avec la poterie funéraire, on l'a vu, incitent davantage à les placer à la fin du $\mathrm{II}^{\mathrm{e}}$ siècle avant $\mathrm{J}$.-C. ou du moins, bien avant la Guerre des Gaules.

Une tentative de rapprochement avec les sites anglais a été faite tout récemment par P. A. Tyers. Les conclusions chronologiques de son étude concernent essentiellement la poterie augustéenne. Sur un plan plus général, il note le parallélisme entre les régions de l'Artois et de Belgique non pas avec le Kent (dont le mobilier est semblable à celui des régions còtières du Nord de la France) mais avec le Yord de la Tamise ${ }^{25}$.

Il semble donc que la classification chronologique du matériel céramique de la région lilloise au $\mathrm{I}^{\text {er }}$ siècle av. J.-(C. soit moins assurée que celle des périodes précédentes; la

24 Voir par exemple le materiel de Villeneuve-Saint-Germain : J. Disond, Premier bilan de huil années de fouilles à Villeneuve-Saint-Germain (Aisne) 19r-30, dans Vallée de T.Aisne, cinq années de fouilles protohisloriques, no spécial 1982, Revue Archénlogique de Picardie, p. 213-264.

25 P. A. Tyers, Correspondance entre la céramique commune de la Tène III du Sud-Est de l'Anglelerre et du Ford de la France, dans Septentrion, 10, 1980, p. 61-70. 
GERMAINE LEMAN-DELERIVE
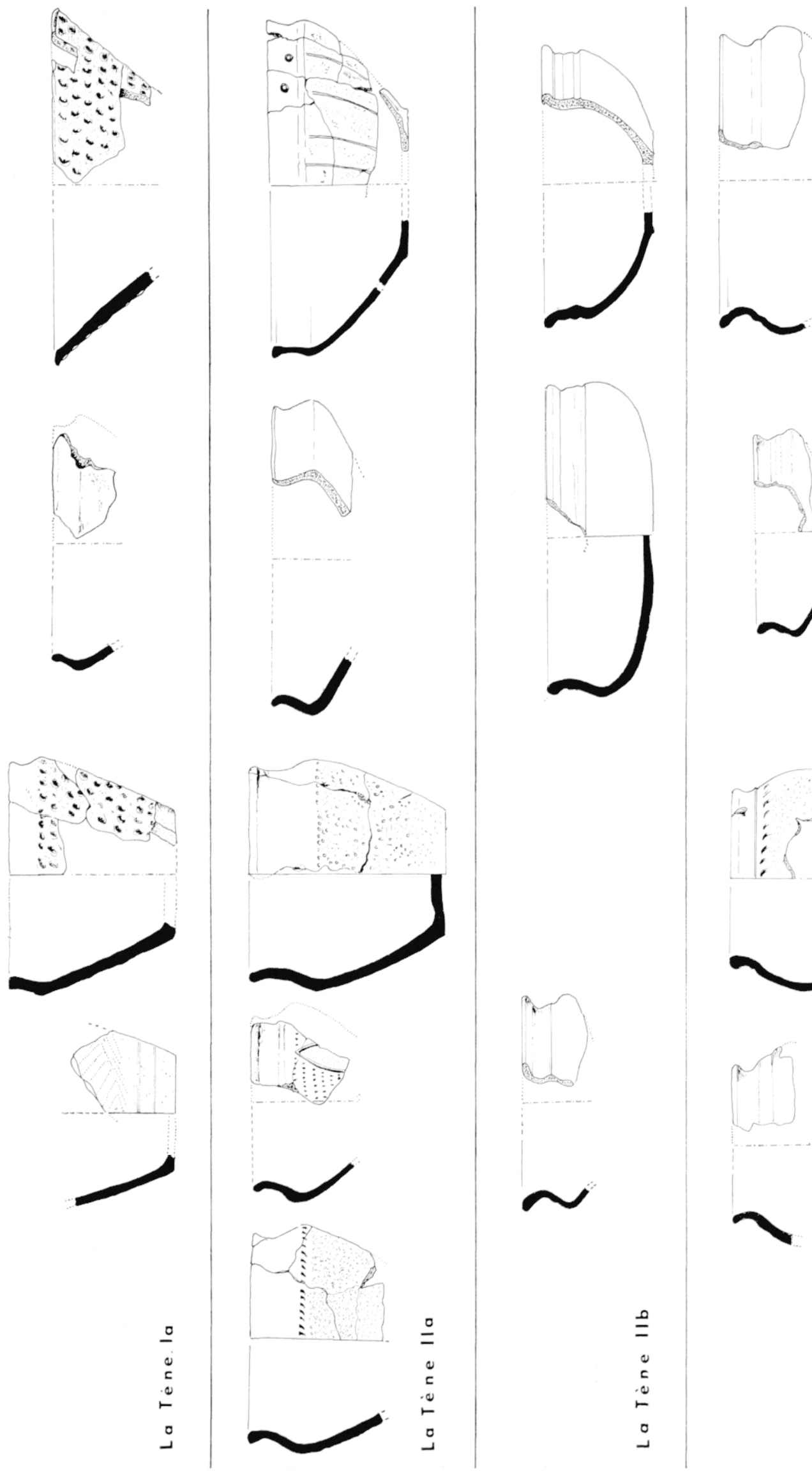

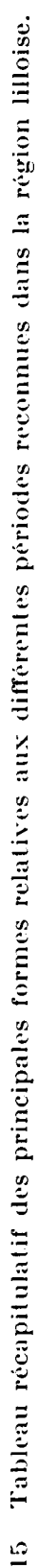
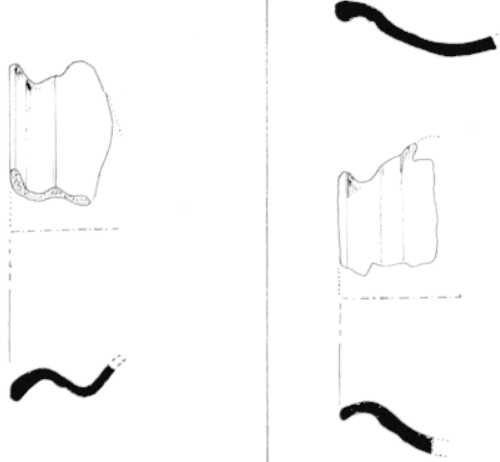

$\stackrel{0}{=}$
0
0
0
0 
datation du mobilier de Loos autour de 100 av. J.-G. est encore à considérer comme une hypothèse de travail.

$$
*
$$

Au cours de l'évolution de cette céramique, documentée à ce jour seulement d'une manière très lacunaire, les techniques d'ornementation ne semblent pas avoir évolué ; à travers ces cinq siècles considérés, les deux grands types de décor sont toujours l'impression au doigt, à l'ongle ou au bâtonnet, et le travail «à l'éclaboussé ». Le décor au peigne est totalement absent.

Si l'on veut tenter une synthèse des résultats par l'analyse chronologique de ces quatre ensembles de céramiques, en les replaçant au sein des autres découvertes plus restreintes de la région lilloise $e^{26}$, il convient d'abord de remarquer une absence totale de vestiges entre le milieu de La Tène I et La Tène moyenne. Le début de cette période semble correspondre à une augmentation du nombre de sites, qui trahit sans doute un accroissement de la population. L'occupation du sol ne diminue pas au début de La Tène finale, mais à nouveau la période post-césarienne est inconnue. Certes, ces observations sont encore bien limitées et ne permettent pas d'induire une conclusion historique, elles constituent néanmoins un premier acquis fort intéressant et fort neuf pour une région si mal connue jusqu'ici (fig. 15).

Par ailleurs, les comparaisons proposées en vue de l'établissement d'une chronologie amènent aussi à considérer les rapports de cette céramique avec celle qui a été exhumée dans le Hainaut tout proche, mais aussi avec les découvertes marniennes. Aussi bien à l'époque de La Tène I qu'à La Tène II et III, les analogies sont frappantes. Peut-être sera-t-on ainsi amené désormais à reconnaître une forme de céramique caractéristique d'un domaine géographique plus vaste qu'on ne le supposait. Dès lors, me semble-t-il, les conclusions élaborées jadis à propos du territoire des Belges, et de leurs prédécesseurs, appellent une révision à court terme.

\section{Germaine Leman-Delerive C.N.R.S.}

26 Il convient d'ajouter les sites non présentés ici en raison du nombre réduit de formes découvertes : pour la Tène ancienne : Mons-en-Barocul (G. Leman-Delerive, Contribution à l'élude de l'Age du fer dans le Nord de la France: structures découvertes à Mons-en-Baroeul dans l'Age du fer en France Septentrionale, dans Mémoires de la Socièté Archéologique Champenoise, 2, 1981, p. 231-234), Seclin, Tourcoing; pour la période de la Tène moyenne et finale : Fàches-Thumesnil, Lille (Pont Royal), Sainghin-en-Mélantois.

N.B. - Il m'est très agréable de remercier ici les personnes qui m'ont tant aidée dans la réalisation de ce travail, en particulier, M. H. Halbaut pour son aide si efficace dans les prospections et les fouilles; M. P. Roualet pour ses nombreux conseils, M. J.-L. Flouest, pour son aimable assistance. M. V. Kruta enfin me guida dans l'orientation de cette recherche : qu'il veuille bien trouver ici l'expression de toute ma gratitude. 